\title{
A SMALL-SCALE FIELD EXPERIMENT FOR THE VALIDATION OF A THEORY ON REFLECTION OF NONLINEAR SHORT-CRESTED WAVE GROUPS
}

\author{
Alessandra Romolo ${ }^{1}$ and Felice Arena ${ }^{2}$
}

\begin{abstract}
A closed-form solution of free surface displacement and velocity potential for nonlinear short-crested (3D) wave groups interacting with a reflective sea wall is presented. The theory is applied to investigate the fluctuation wave pressures when an exceptionally high crest or deep trough occurs on the reflective wall. A characteristic behaviour has been observed. Due to non-linearity, on one hand, great reduction of the highest crest and enhancement of the deepest troughs on wave pressures are realized; on the other hand, the profiles show always a strong asymmetry between the absolute maxima and the minima and sometimes the formation of characteristic humped wave pressures corresponding to the impact on the structure of the highest wave crest. All the theoretical results have been validated through a smallscale field experiment carried out at the Natural Ocean Engineering Laboratory (N.O.E.L., www.noel.unirc.it) of Reggio Calabria (Italy).
\end{abstract}

Keywords: nonlinear sea wave, reflection, short-crested wave groups, wave pressure, sea wall, upright breakwater

\section{INTRODUCTION}

One of the most basic issues of coastal engineering has been the estimation of the wave pressures and wave loads acting on a vertical sea wall.

In the scientific literature, it is possible to find several theories and models, based on different approaches, formulated with the aim, on one hand, to describe as the most correctly as possible the wave mechanics of sea waves interacting with a reflective structure in the absence of overtopping and without considering breaking waves generating impulsive pressure, and on the other hand, to find the most accurate solution for the calculation of wave pressure distribution acting on the structure for practical engineering applications. Many experimental investigations have been also conducted to validate theoretical solutions.

First order solutions of periodic or irregular waves, are important since they are the basic approach for the prediction of wave pressure. More accurate results turn out by considering higher order contributions. Through the periodic wave theory, solution of second-order was obtained by Saintflou (1928), and by Svendsen and Jonsson (1976); of third-order by Tadjbakhsh \& Keller (1960); of fourthorder by Goda $(1967,1999)$.

Moreover, numerical models have been developed to validate analytical results and to investigate effects of higher order components. Numerical solutions with perturbation approximations up to thirdorder in wave steepness have been given by Jeng (2002), up to the 27th order by Roberts $(1983 a, b)$ and up to the 35th order by Marchant and Roberts (1987). Numerical solutions with Fourier approximation for short-crested waves up to 10th order have been derived by Roberts and Schwartz (1983), by Lin et al. (1987) and by Tsai et al. (1994).

More recent numerical study with experimental comparison have been done by Kimmoun et al. (1999) and by Prabhkar and Sundar (2001).

The problem of instability for the reflection of sea waves with the related effects and phenomenon was dealt, in the last years, by Mercer and Roberts (1992, 1994), Schultz et al. (1998), LonguetHiggins and Dommermuth (2001a,b), Longuet-Higgins and Drazen (2002) and Peregrine (2003).

The linear Quasi-Determinism (QD) theory of the highest waves (Boccotti 1981, 1982, 1989, 1997, 2000), which is able to describe the mechanics of three-dimensional sea wave groups, associated to the finite bandwidth of wave spectrum, has been applied by Boccotti to investigate the mechanics of linear sea wave groups in reflection $(1988,1997,2000)$ and validated by himself through small-scale field experiments carried out in the natural laboratory of Reggio Calabria (1993b, 1997, 2000).

The correction up to second-order of the linear solution of wave groups in reflection, achieved through the QD theory, has been derived by Romolo (2007) and described in Romolo and Arena for both long-crested wave groups (2D) (2007-2008a) and short-crested (3D) wave groups (2008b). The proposed solution points out as non-linearity strongly affects the linear predictions both in the timespace evolution of the sea groups in reflection and in the time fluctuations of wave pressures and, thus,

\footnotetext{
${ }^{1}$ Mediterranea' University, Faculty of Engineering, Dept. MECMAT, Loc. Feo di Vito, 89122, Reggio Calabria, ITALY; aromolo@unirc.it

${ }^{2}$ Mediterranea' University, Faculty of Engineering, Dept. MECMAT, Loc. Feo di Vito, 89122, Reggio Calabria, ITALY; arena@unirc.it
} 
in the wave force acting on the structure, when wave groups with very high crests or deep troughs impact the upright fully reflective structure. Interesting results have been outlined and will be described in the paper, which concerns also on the validation of the theoretical prediction with the data of a small-scale field experiment, carried out in May 2009 in the Natural Ocean Engineering Laboratory (N.O.E.L., www.noel.unirc.it) of the Mediterranea University of Reggio Calabria, Italy.

\section{ANALYTICAL MODEL}

\section{Governing equations}

In this study the wave field resulting from short-crested (three-dimensional) wave groups interacting with a vertical sea wall and fully reflected by it, is considered.

The fluid is assumed inviscid, incompressible and the flow irrotational in a constant depth. Thus the hydrodynamic equations governing the problem to the second-order with the two nonlinear boundary conditions are

the continuity equation (Laplace's equation):

$$
\nabla^{2} \bar{\phi}_{2_{R}}=0 \quad \text { for }-d \leq z \leq \bar{\eta}_{R}=\bar{\eta}_{1_{R}}+\bar{\eta}_{2_{R}},
$$

the Kinematics Free Surface Boundary Condition (KFSBC):

$$
\partial_{T} \bar{\eta}_{2_{R}}-\partial_{z} \bar{\phi}_{2_{R}}=H(X, Y, z, T) \quad \text { at } z=0,
$$

the Dynamic Free Surface Boundary Condition (DFSBC):

$$
g \bar{\eta}_{2_{R}}+\partial_{T} \bar{\phi}_{2_{R}}=K(X, Y, z, T) \quad \text { at } z=0,
$$

the Bottom Boundary Condition (BBC):

$$
\partial_{z} \bar{\phi}_{2_{R}}=0 \quad \text { at } z=-d,
$$

and the Wall Boundary Condition (WBC):

$$
\partial_{Y} \bar{\phi}_{2_{R}}=0 \quad \text { at } Y=-y_{0} \text { with } y_{0} \leq 0
$$

where

$$
\begin{aligned}
& \left.H(X, Y, z, T)\right|_{z=0}=-\left(\partial_{X} \bar{\phi}_{1_{R}}\right)\left(\partial_{X} \bar{\eta}_{1_{R}}\right)-\left(\partial_{Y} \bar{\phi}_{1_{R}}\right)\left(\partial_{Y} \bar{\eta}_{1_{R}}\right)+\left(\partial_{z z} \bar{\phi}_{1_{R}}\right) \bar{\eta}_{1_{R}}, \\
& \left.K(X, Y, z, T)\right|_{z=0}=-\left(\partial_{z T} \bar{\phi}_{1_{R}}\right) \bar{\eta}_{1_{R}}-\frac{1}{2}\left[\left(\partial_{X} \bar{\phi}_{1_{R}}\right)^{2}+\left(\partial_{Y} \bar{\phi}_{1_{R}}\right)^{2}+\left(\partial_{z} \bar{\phi}_{1_{R}}\right)^{2}\right],
\end{aligned}
$$

define the Quadratic Transfer Functions (QTFs), respectively, of the first-order velocity potential and of the linear free surface displacement and their partial derivatives.

In all the expressions $[(1) \div(7)]$, the subscripts denote the partial differentiation.

The complexity to obtained a closed-form solution for both $\bar{\eta}_{2_{R}}$ and $\bar{\phi}_{2_{R}}$ is just in the analytical resolution of the two Quadratic Transfer Functions (6) and (7).

\section{Solution of $\bar{\eta}_{1_{R}}$ and $\bar{\phi}_{1_{R}}$ through the linear Quasi-Determinism theory}

The linear Quasi-Determinism (QD) theory, formulated by Boccotti (1981, 1982, 1989, 1997 , 2000), allows for the achievement, under specific assumption, of an expected configuration in space and time of both the free surface displacement and the velocity potential.

If a very high wave crest, of elevation $H_{C}$, occurs at a fixed point $\underline{x}_{0}$ at a given time instant $t_{0}$, in a stationary Gaussian process, like a wind-generated sea state, through the QD the expressions of the free surface and of the velocity potential tend, with very high probability, to closed-forms in the limit $H_{C} / \sigma \rightarrow \infty$ ( $\sigma$ being the standard deviation of the wave field where $H_{C}$ is realized).

The deterministic surface elevation is

$$
\bar{\eta}\left(\underline{x}_{0}+\underline{X}, t_{0}+T\right)=H_{C} \frac{\Psi(\underline{X}, T)}{\Psi(\underline{0}, 0)}
$$

and the velocity potential is

$$
\bar{\phi}\left(\underline{x}_{0}+\underline{X}, z, t_{0}+T\right)=H_{C} \frac{\Phi(\underline{X}, z, T)}{\Psi(\underline{0}, 0)} .
$$

with $\Psi(\underline{X}, T)$ and $\Phi(\underline{X}, T)$ respectively the autocovariance of the surface displacement and the crosscovariance of the surface displacement and the velocity potential, defined as 


$$
\begin{aligned}
& \Psi(\underline{X}, T)=<\eta\left(\underline{x}_{0}, t\right) \eta\left(\underline{x}_{0}+\underline{X}, t+T\right)>, \\
& \Phi(\underline{X}, z, T)=<\eta\left(\underline{x}_{0}, t\right) \phi\left(\underline{x}_{0}+\underline{X}, z, t+T\right)>,
\end{aligned}
$$

where $\eta$ and $\phi$ are stationary Gaussian processes.

That one described is the first formulation of the QD theory (Boccotti 1981, 1982, 1989, 2000). Expressions (8) and (9) are exact to the first order in a Stokes expansion and, above all, hold for nearly arbitrary bandwidth and solid boundary, as proved by Boccotti (2000).

The solution of the linear deterministic functions (8) and (9) for the reflection of short-crested (3D) wave groups was achieved by Boccotti $(1988,1993 b, 1997,2000)$ giving the following equations for the free surface displacement and the velocity potential as a function of the directional wave spectrum $S(\omega, \theta)$ of the incident waves:

$$
\begin{aligned}
& \bar{\eta}_{1_{R}}\left(\underline{x}_{0}+\underline{X}, t_{0}+T\right)=\frac{H_{C_{R}}}{\sigma^{2}} \int_{0}^{\infty} \int_{0}^{2 \pi} S\left(\omega_{1}, \theta_{1}\right) \cos \left(\lambda_{1}\right) \cos \left(\varphi_{1}\right) \cos \left(\alpha_{1}\right) \mathrm{d} \theta_{1} \mathrm{~d} \omega_{1} \\
& \bar{\phi}_{1_{R}}\left(\underline{x}_{0}+\underline{X}, z, t_{0}+T\right)=\frac{g H_{C_{R}}}{\sigma^{2}} \int_{\substack{0 \\
\omega_{1}}}^{\infty} \int_{0}^{2 \pi} S\left(\omega_{1}, \theta_{1}\right) \omega_{1}^{-1} \frac{\cosh \left[k_{1}(d+z)\right]}{\cosh \left(k_{1} d\right)} \sin \left(\lambda_{1}\right) \cos \left(\varphi_{1}\right) \cos \left(\alpha_{1}\right) \mathrm{d} \theta \mathrm{d} \omega \\
& \text { where } \\
& \varphi_{n}\left(k_{n}, \theta_{n}, y_{0}\right)=k_{n} y_{0} \cos \theta_{n} \\
& \alpha_{n}\left(k_{n}, \theta_{n}, y_{0}, Y\right)=k_{n} \cos \theta_{n}\left(y_{0}+Y\right) \quad n=1, \\
& \lambda_{n}\left(k_{n}, \theta_{n}, X, T\right)=k_{n} \sin \theta_{n} X-\omega_{n} T
\end{aligned}
$$

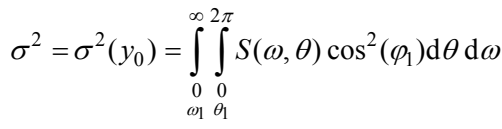

with wave frequency, $\omega$, and wavenumber, $k$, satisfying the dispersion relation

$$
\omega_{1}^{2}=g k_{1} \tanh \left(k_{1} d\right) \text {. }
$$

From the Bernoulli's equation, the linear wave pressure acting on the wall is calculated

$$
\begin{aligned}
& \bar{p}_{w_{1_{R}}}\left(\underline{x}_{0}+\underline{X}, z, t_{0}+T\right)= \\
& \frac{\rho g H_{C_{R}}}{\sigma^{2}} \int_{0}^{\infty} \int_{0}^{2 \pi} S\left(\omega_{1}, \theta_{1}\right) \frac{\cosh \left[k_{1}(d+z)\right]}{\cosh \left(k_{1} d\right)} \cos \left(\lambda_{1}\right) \cos \left(\varphi_{1}\right) \cos \left(\alpha_{1}\right) \mathrm{d} \theta \mathrm{d} \omega .
\end{aligned}
$$

Equations (12), (13) and (17) are referred to the frame of reference depicted in Figure 1.

\section{Solution of the second-order components $\bar{\eta}_{2_{R}}$ and $\bar{\phi}_{2_{R}}$}

Starting from the equations (12) and (13) of $\bar{\eta}_{1_{R}}$ and $\bar{\phi}_{1_{R}}$, the Quadratic Transfer Functions (6) and (7), governing the examined problem, have been derived. The line of reasoning is the same of that proved in Romolo (2007) and Romolo and Arena (2007, 2008a) for long-crested (two-dimensional) wave groups. Initial results were shown in Romolo and Arena (2008b).

The complete solution for $\bar{\eta}_{2_{R}}$ and $\bar{\phi}_{2_{R}}$ is

$$
\begin{aligned}
& \bar{\eta}_{2_{R}}\left(\underline{x}_{0}+\underline{X}, t_{0}+T\right)=\frac{H_{C_{R}}^{2}}{8 \sigma^{4}} \int_{0}^{\infty} \int_{0}^{\infty} \int_{0}^{\infty} \int_{0}^{2 \pi} \int_{0}^{2 \pi} S\left(\omega_{1}, \theta_{1}\right) S\left(\omega_{2}, \theta_{2}\right) \cos \left(\varphi_{1}\right) \cos \left(\varphi_{2}\right) \\
& \cdot\left\{\left[\mathrm{A}_{1}^{-} \cos \left(\alpha_{1}-\alpha_{2}\right)+\mathrm{A}_{2}^{-} \cos \left(\alpha_{1}+\alpha_{2}\right)\right] \cos \left(\lambda_{1}-\lambda_{2}\right)+\left[\mathrm{A}_{1}^{+} \cos \left(\alpha_{1}+\alpha_{2}\right)\right.\right. \\
& \left.\left.+\mathrm{A}_{2}^{+} \cos \left(\alpha_{1}-\alpha_{2}\right)\right] \cos \left(\lambda_{1}+\lambda_{2}\right)\right\} \mathrm{d} \theta_{2} \mathrm{~d} \theta_{1} \mathrm{~d} \omega_{2} \mathrm{~d} \omega_{1}-\Xi / g
\end{aligned}
$$



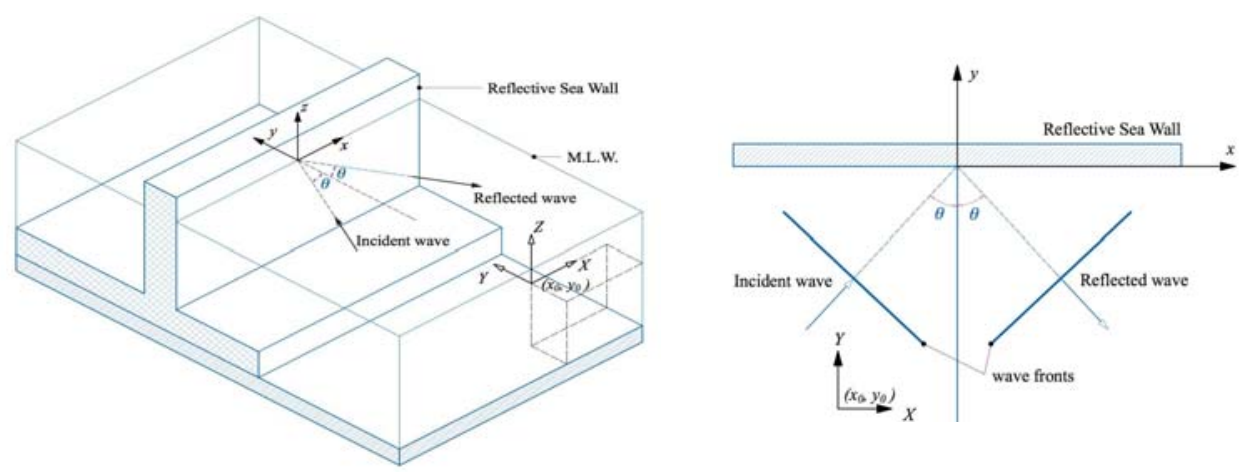

Figure 1. A sketch diagram of the considered three-dimensional wave motion produced by sea wave groups interacting with a vertical sea wall: the tridimensional cross section (a) and the plan (b) are depicted. The absolute Cartesian coordinate system $(\underline{x}, z)=(x, y, z)$ is fixed at the structure and a relative Cartesian coordinate system $\left(\underline{x}_{0}, z\right)=\left(x_{0}, y_{0}, 0\right)$ at point where the exceptionally either high crest or trough amplitude occurs, which could be at or in front of the structure.

$$
\begin{aligned}
& \bar{\phi}_{2_{R}}\left(\underline{x}_{0}+\underline{X}, z, t_{0}+T\right)= \\
& g^{2} \frac{H_{C_{R}}^{2}}{8 \sigma^{4}} \int_{\substack{0 \\
\omega_{1} \omega_{2}}}^{\infty} \int_{0}^{\infty} \int_{0}^{2 \pi} \int_{0}^{2 \pi} S\left(\omega_{1}, \theta_{1}\right) S\left(\omega_{2}, \theta_{2}\right) \omega_{1}^{-1} \omega_{2}^{-1} \cos \left(\varphi_{1}\right) \cos \left(\varphi_{2}\right) \\
& \cdot\left\{\left[\mathrm{C}_{1}^{-} \cos \left(\alpha_{1}-\alpha_{2}\right)+\mathrm{C}_{2}^{-} \cos \left(\alpha_{1}+\alpha_{2}\right)\right]\left(\omega_{1}-\omega_{2}\right)^{-1} \sin \left(\lambda_{1}-\lambda_{2}\right)+\left[\mathrm{C}_{1}^{+} \cos \left(\alpha_{1}+\alpha_{2}\right)\right.\right. \\
& \left.\left.+\mathrm{C}_{2}^{+} \cos \left(\alpha_{1}-\alpha_{2}\right)\right]\left(\omega_{1}+\omega_{2}\right)^{-1} \sin \left(\lambda_{1}+\lambda_{2}\right)\right\} \mathrm{d} \theta_{2} \mathrm{~d} \theta_{1} \mathrm{~d} \omega_{2} \mathrm{~d} \omega_{1}+\Xi T
\end{aligned}
$$

$H_{C R}$ is the exceptionally linear high crest elevation, $\sigma$ is expressed by relation (15), and $\mathrm{A}_{n}^{\mp}$ and $\mathrm{C}_{n}^{\mp}$ $(n=1,2)$ parameters are the so called interaction kernels respectively of the nonlinear free surface and of the velocity potential related to their quadratic transfer functions (the expressions are reported in the Appendix). $S\left(\omega_{n}, \theta_{n}\right)(n=1,2)$ is the directional wave spectrum of the incident waves. $\phi_{n}, \alpha_{n}, \lambda_{n}(n=1,2)$ are defined by relations (14).

Retaining the terms up to the second-order by the Bernoulli's equation, the nonlinear deterministic wave pressure is achieved

$$
\begin{aligned}
& \bar{p}_{w_{2_{R}}}\left(\underline{x}_{0}+\underline{X}, z, t_{0}+T\right)=\rho g^{2} \frac{H_{C_{R}}^{2}}{2 \sigma^{4}}\left\{\frac{1}{4} \int_{\substack{0 \\
\omega_{1} \omega_{2}}}^{\infty} \int_{0}^{\infty} \int_{0}^{\infty} \int_{0}^{2 \pi} S\left(\omega_{1} \theta_{1}, \theta_{1}\right) S\left(\omega_{2}, \theta_{2}\right) \omega_{1}^{-1} \omega_{2}^{-1} \cos \left(\varphi_{1}\right)\right. \\
& \cdot \cos \left(\varphi_{2}\right)\left\{\left\{\mathrm{C}_{1}^{-} \cos \left(\alpha_{1}-\alpha_{2}\right)+\mathrm{C}_{2}^{-} \cos \left(\alpha_{1}+\alpha_{2}\right)\right\} \cos \left(\lambda_{1}-\lambda_{2}\right)+\left\{\mathrm{C}_{1}^{+} \cos \left(\alpha_{1}+\alpha_{2}\right)+\mathrm{C}_{2}^{+}\right.\right. \\
& \left.\left.\cdot \cos \left(\alpha_{1}-\alpha_{2}\right)\right\} \cos \left(\lambda_{1}+\lambda_{2}\right)\right\} \mathrm{d} \theta_{2} \mathrm{~d} \theta_{1} \mathrm{~d} \omega_{2} \mathrm{~d} \omega_{1}-\sum_{p=1}^{3}\left[\int_{0}^{\infty} \int_{0}^{2 \pi} S\left(\omega_{1}, \theta_{1}\right) \frac{\omega_{1}^{-1} k_{1}}{\cosh \left(k_{1} d\right)} \cos \left(\varphi_{1}\right)\right. \\
& \left.\left.\cdot \mathrm{Q}_{p}\left(\underline{x}_{0}+\underline{X}, z, t_{0}+T ; \omega_{1}, \theta_{1}\right) \mathrm{d} \theta_{1} \mathrm{~d} \omega_{1}\right]^{2}\right\}-\rho \Xi ; \quad \text { with } p=1,2,3 \\
& \mathrm{Q}_{p}\left(\underline{x}_{0}+\underline{X}, z, t_{0}+T ; \omega_{1}, \theta_{1}\right)=\left\{\begin{array}{cc}
\cosh \left[k_{1}(d+z)\right] \sin \left(\theta_{1}\right) \cos \left(\alpha_{1}\right) \cos \left(\lambda_{1}\right) & \text { for } p=1 \\
-\cosh \left[k_{1}(d+z)\right] \cos \left(\theta_{1}\right) \sin \left(\alpha_{1}\right) \sin \left(\lambda_{1}\right) & \text { for } p=2 \\
\sinh \left[k_{1}(d+z)\right] \cos \left(\alpha_{1}\right) \sin \left(\lambda_{1}\right) & \text { for } p=3
\end{array}\right. \text {. }
\end{aligned}
$$

In solutions (18), (19) and (21), the parameter $\Xi$ is expressed by relation

$$
\Xi=-g \frac{H_{C_{R}}^{2}}{8 \sigma^{4}} \int_{\substack{0 \\ \omega_{1}}}^{\infty} \int_{\substack{\omega_{2} \\ \theta_{1}}}^{\infty} \int_{0}^{2 \pi 2 \pi} \int_{\substack{0 \\ \theta_{2}}}^{2 \pi} S\left(\omega_{1}, \theta_{1}\right) S\left(\omega_{2}, \theta_{2}\right) F\left(\omega_{1}, \omega_{2}, \theta_{1}, \theta_{2}\right) \mathrm{d} \theta_{2} \mathrm{~d} \theta_{1} \mathrm{~d} \omega_{2} \mathrm{~d} \omega_{1}
$$

where

$$
F\left(\omega_{1}, \omega_{2}, \theta_{1}, \theta_{2}\right)= \begin{cases}\frac{2 k_{1}}{\sinh \left(2 k_{1} d\right)} \cos ^{2}\left(\varphi_{1}\right) & \text { if } \omega_{1}=\omega_{2} \text { and } \theta_{1}=\theta_{2} \\ 0 & \text { if } \omega_{1} \neq \omega_{2} \quad \text { and } \theta_{1} \neq \theta_{2}\end{cases}
$$




\section{SMALL-SCALE FIELD EXPERIMENT IN THE NATURAL OCEAN ENGINEERING LABORATORY (N.O.E.L.) OF REGGIO CALABRIA, ITALY.}

\section{General description of the experiment}

The laboratory of ocean engineering N.O.E.L. (www.noel.unirc.it) is located on the waterfront of Reggio Calabria in the East coast of the Strait of Messina. Thanks to some exceptional natural conditions of the site, in the laboratory it is possible to carry out experiments directly in the sea operating through the techniques of the laboratory tanks. The feasibility of these procedures and the effectiveness of the results were explained and verified in detail by Boccotti (Boccotti et al. 1993a, Boccotti 1997, 2000).

In May 2009, an experiment to study the interaction of sea wave groups with a vertical sea wall was carried out in the N.O.E.L. laboratory. The feature of the experiment is reported in Figure 2. The structure is a small upright breakwater with the frame in reinforced concrete, with a length of $16.4 \mathrm{~m}$, a height of $3.0 \mathrm{~m}$ and placed in a depth of $1.9 \mathrm{~m}$ with respect to the MWL (mean water level).

The fluctuating wave pressures acting on the sea wall were measured by 16 pressure transducers, placed, in the sea beaten side, along the cross-section of the central caisson making the breakwater.

The incident waves (in the undisturbed field), not influenced by the presence of the structure, were measured by means of two ultrasonic probes and two pressure transducers. One of each instrument was assembled on a thin pile (diameter of $0.05 \mathrm{~m}$ ), located $20 \mathrm{~m}$ far from the sea wall. The configuration is reported in Figure 2.

The local wind of Reggio Calabria often generated waves, which consist of pure wind waves without swell components, with significant wave height ranging among $0.20 \mathrm{~m}<H_{S}<0.40 \mathrm{~m}$ and peak period among $1.8 s<T_{p}<2.6 s$. That represent, in the hydraulic Froude similitude, the scale model of severe storms. Moreover, the wave spectra are very typical of wind wave spectra.

During the experiment of May 2009, 95 sea states of pure wind-waves were recorded with frequency sampling of $10 \mathrm{~Hz}$, each having duration equal to five minutes.

The significant wave height, in the undisturbed field, in the analysed record was between $0.21 \mathrm{~m}$ and $0.41 \mathrm{~m}$, and the peak period between $1.9 \mathrm{~s}$ and $2.74 \mathrm{~s}$; thus the relative water depth was such that $0.16 \leq d / L_{\mathrm{p} 0} \leq 0.32$. Because of the excursion of the water depth $d$ ranged between $1.76 \mathrm{~m}$ and $1.95 \mathrm{~m}$. The dominant wave direction, $\theta_{d o m}$, ranged in $\left[-9^{\circ} ; 1^{\circ}\right]$. The sign of $\theta_{d o m}$ is represented in Figure 2.

For the analyses carried out in this study, only the records of pure wind waves have been considered, with spectra similar to a mean JONSWAP-Mitsuyasu (Hasselmann et al., 1973; Mitsuyasu et al., 1975). More details of the experiment are given in Boccotti et al. (2010).
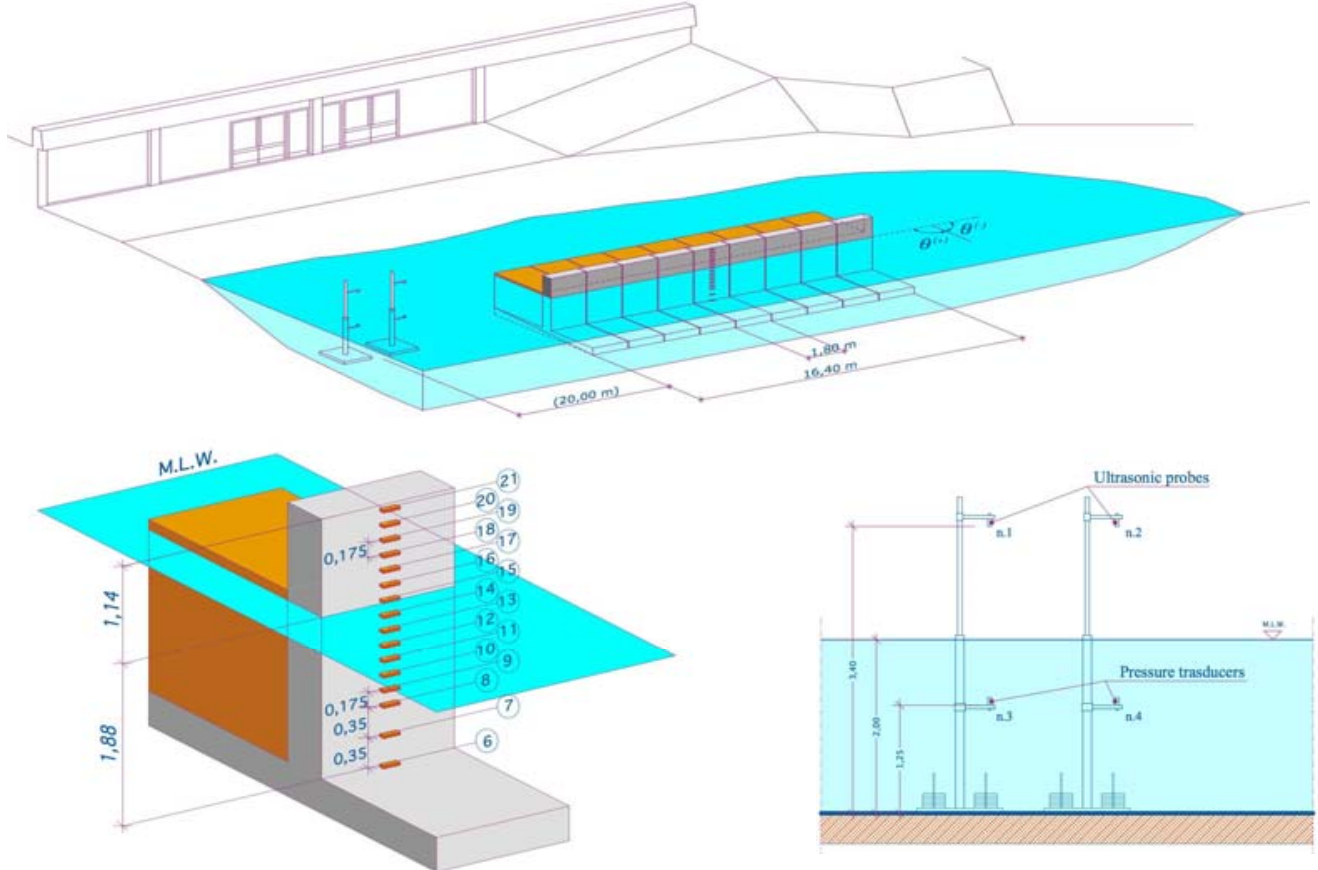

Figure 2. The scheme of the field experiment executed on the sea of Reggio Calabria in May 2009. Threedimensional plan and section of the reflective upright structure utilized for the experiment, map of the gauges at the structure and on the piles in undisturbed field. 


\section{COMPARISON BETWEEN THE PROPOSED NONLINEAR WAVE THEORY FOR THE REFLECTION OF SEA WAVE GROUPS WITH DATA OF THE SMALL-FIELD EXPERIMENT Theoretical results}

The proposed nonlinear solution is able to describe the space-time evolution of nonlinear sea wave groups propagating in front of a vertical sea wall and interacting with it, when a very high wave crest, $H_{C R}$, is realized on the structure or in front of it (Romolo 2007).

Considering the configuration associated to the occurrence of the highest wave crest at the time instant $T=0$ at the structure $\left(y_{0}=0\right)$, in Figure 3, the linear and the nonlinear wave pressures in time domain at different water depth along the cross section of an upright wall are represented.

The non-linearity on the water surface produces an increment of the highest wave crests and a reduction of the deepest sea troughs. An effect which increases when the relative water depth $\left(d / L_{\mathrm{p} 0}\right)$ decreases. A complete survey of these results is in Romolo (2007) and, for long-crested wave groups, in Romolo and Arena (2008a).

An opposite behaviour is observed on the fluctuating wave pressure. On the wave pressure, the non-linearity reduces the highest crest elevations and enhances the deepest trough depths. That is because a minimum of the second-order component is found to be in phase with both the linear highest crest and the deepest troughs, as it can be well-appreciated on Figure 3.

A further important result comes out from the theoretical nonlinear solution. In the time domain, the pressure fluctuations show profiles with strong asymmetries, with the amplitude of the deepest wave trough markedly exceeding the elevation of the highest wave crest. This feature turns out to be enhanced at the bottom depth with respect to mean level water.

For i.e., for the case represented in Figure 3, the ratio between the amplitudes of the maximum crest and the minimum trough of the nonlinear wave pressure is equal to 0.69 at depth $z / d=-0.26$ along the cross-section of the structure; 0.62 at $z / d=-0.53$ and 0.60 at $z / d=-1.00$.

Moreover, moving from the water surface to the bottom depth, another important feature in time variation of the wave pressure becomes evident, this is a distortion on the crest profile with the occurrence of characteristic humped wave pressures. At time instant $T=0$, when the theory expects the occurrence of the highest wave crest, a local minimum of the pressure may be observed with the formation of two pressure maxima for each wave crest impact (see Figure 3).

The formation or not of the characteristic humped wave pressures could be well understood by analysing all the contributions defining the second-order wave pressure. The $\bar{p}_{w_{2 R}}$ [Eq. (20)] is given by a constant term proportional to the constant $\Xi$ of Eq. (22) [term (e) of Figure 4], the terms due to $\bar{\phi}_{2_{R}}$ and that due to $\bar{\phi}_{1_{R}}$ [term (c) of Figure 4]. Those derived by $\bar{\phi}_{2_{R}}$ are the positive and the negative kernels of interaction respectively proportional to the parameters $\mathrm{C}_{n}^{-}$and $\mathrm{C}_{n}^{+}(n=1,2)$ with $\omega_{1} \neq \omega_{2}$ and $\theta_{1} \neq \theta_{2}$ [respectively, the term (b) and the (a) of Figure 4] and the self-interaction term [term (d) of Figure 4] proportional to the parameters $C_{n}^{\mp}(n=1,2)$ with $\omega_{1}=\omega_{2}$ and $\theta_{1}=\theta_{2}$.

In Figure 4 (for the case represented in Figure 3), we have analysed the trend of all the terms previously defined at the relative water depth, $z / d,-1$ and -0.16 . As we can observe, the presence or not of the characteristic humped wave pressures is essentially due to the combined behaviour of the positive interaction kernels and of the term proportional to the linear potential. At the sea bottom $(z / d=$ -1) the nonlinear term associated to $\bar{\phi}_{1_{R}}$ does not give contribution. Instead, the positive kernels show double maxima under the linear highest crest producing double positive peaks. Going towards the water surface $(z / d=-0.16)$, on one hand, the trend of positive kernels keeps constant reducing in amplitude; on the other hand, the term derived by the linear potential produces two minima just in the correspondence of the two maxima of the positive kernels becoming of the same order of magnitude at the mean level water. These effects explain the progressive disappearance of the humped wave pressure profiles.

All the described properties are greatly influenced by the water depth and wave conditions, by which every phenomenon could be enhanced or reduced. 


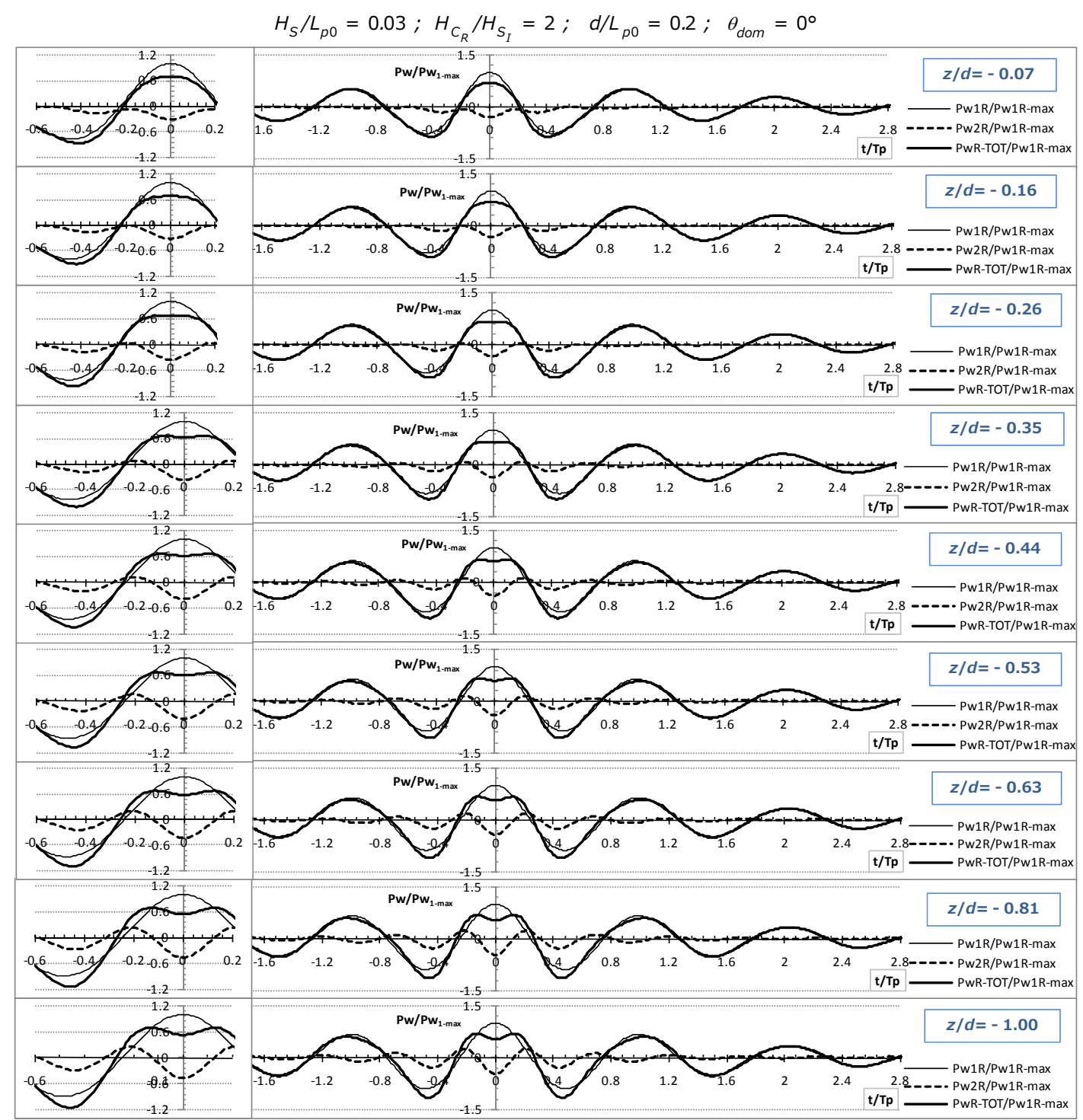

Figure 3. Theoretical linear and nonlinear fluctuating wave pressures acting on the reflective sea wall, when an exceptionally high crest elevation $H_{C R}$ is realized at time instant $T=0$ on the structure $\left(y_{0}=0\right)$.

\section{Validation of the theory with experimental data}

First of all, all the records of the experiment have been analysed to single out those with the occurrence of the overtopping waves and those without overtopping (see Boccotti et al., 2010). In the present paper only the records without the occurrence of overtopping have been considered.

In Figure 5, the time series recorded by pressure transducers 6, 11 and 16 of the record 951 are shown. As we can see, in the correspondence to the wave crests at the structure (that are identified by the signal of the upper pressure transducers), we recognise, almost always, the formation of characteristic humped wave pressures in the deeper instrument. Similar trends were well observed and described by Peregrine (2003).

From the measured wave pressures, by simple integration along depth, the wave forces acting on the structure can be computed.

As for the theory, it can be applied when an exceptionally linear high crest elevation $H_{C R}$, or an exceptionally high trough amplitude $H_{T R}$, is realized at the reflective structure $\left(y_{0}=0\right)$ or in front of it $\left(y_{0}<0\right)$. This implies that the nonlinear water elevation will be evaluated as

$$
\bar{\eta}_{R}\left(\underline{x}_{0}+\underline{X}, z, t_{0}+T\right)=\bar{\eta}_{1_{R}}\left(\underline{x}_{0}+\underline{X}, z, t_{0}+T\right)+\bar{\eta}_{2_{R}}\left(\underline{x}_{0}+\underline{X}, z, t_{0}+T\right),
$$

by considering the occurrence of an exceptionally linear high crest elevation $H_{C R}$, and as

$$
\bar{\eta}_{R}\left(\underline{x}_{0}+\underline{X}, z, t_{0}+T\right)=-\bar{\eta}_{1_{R}}\left(\underline{x}_{0}+\underline{X}, z, t_{0}+T\right)+\bar{\eta}_{2_{R}}\left(\underline{x}_{0}+\underline{X}, z, t_{0}+T\right),
$$


by considering the occurrence of an exceptionally high linear trough amplitude $H_{T R}$. Same laws regulate also the nonlinear velocity potential and the nonlinear wave pressure.

In the applications we have considered only the case when the very high elevation, $H_{C R}$ or $H_{T R}$, is realized at the structure, that is to say $y_{0}=0$, since it is more interesting for practical engineering applications. The theory has been applied always by considering all the characteristic parameters defining the undisturbed wave field, like the significant wave height, $H_{\mathrm{S}}$, the peak period, $T_{\mathrm{p}}$, the tide level, тL, the dominant wave direction, $\theta_{d o m}$, and the relative water depth, $d / L_{\mathrm{p} 0}$, equal to those of the analysed record. The analytical directional spectrum has been a JONSWAP mean- Mitsuyasu. For the spectra bandwidth the Boccotti's $\Psi^{*}$ parameter (Boccotti, 2000) is calculated for each sea states, $\Psi^{*}$ being the quotient between the absolute value of the first minimum and maximum of autocovariance function. It ranges between 0 and 1 , it is equal to 1 for an infinitely narrow spectrum and equals 0.73 for the mean JONSWAP spectrum.

$H_{C R}$ or $H_{T R}$ are assumed equal to eight times the standard deviation of the recorded water elevation in undisturbed wave field.

The record 951 is characterized by the following values $H_{\mathrm{S}}=0.31 \mathrm{~m}, T_{\mathrm{p}}=2.2 \mathrm{~s}, \quad \mathrm{TL}_{\mathrm{L}}=-0.05 \mathrm{~m}$, $\theta_{\text {dom }}=3^{\circ}, d / L_{\mathrm{p} 0}=0.24, \Psi^{*}[z=0]=0.67, \Psi^{*}[z=-0.60 \mathrm{~m}]=0.82, \Psi^{*}[z=-1.90 \mathrm{~m}]=0.82$. That is the record with the occurrence of the maximum, in absolute value, wave force of the experiment, which corresponds to the negative peak of the wave force $F_{w}$ of the record. In value, it is equal to $4.48 \sigma_{F w}, \sigma_{F w}$ being the standard deviation of the wave force process $F_{w}$.

Instead, in record 1002, characterized by the values: $H_{\mathrm{S}}=0.312 \mathrm{~m}, T_{\mathrm{p}}=2.34 \mathrm{~s}$, тL $=-0.052 \mathrm{~m}, \theta_{\text {dom }}=8^{\circ}$, $d / L_{\mathrm{p} 0}=0.208, \Psi *[z=0]=0.65, \Psi^{*}[z=-0.60 \mathrm{~m}]=0.87, \Psi^{*}[z=-0.60 \mathrm{~m}]=0.86$, the maximum positive peak of the wave force is realized, which is equal to $4.09 \sigma_{F w}$.

The comparisons between the theoretical and the experimental wave pressure profiles are shown in Figure 6 for the record 951, and in Figure 7 for the record 1181.

For the record 951 (Figure 6) the theory has been applied with the occurrence of a very deep wave trough at time instant $t / T_{p}=0$ (Eq. 25). For the record 1181 (Figure 7), we have considered the occurrence an exceptionally high crest amplitude at time instant $t / T_{p}=0$ (Eq. 24). The theory seems to match well with the experimental data in terms both of values and of trends. Characteristic behaviours have been indentified in both cases.

A strong non-linearity in wave pressures has been observed, with a time shift between the crest of the free surface displacement (which is associated to the pressures measured by the highest pressure transducers) and the corresponding crest of the wave pressures measured by the deepest pressure transducers.

In detail, we have, from Figure 7, the crest of the $\bar{\eta}_{R}$ process at sea wall and the crest of pressure transducer 6 with time shift $-0.12 T_{p}$. Also in Figure 6 we find that, the crests before and after the deepest wave trough suffer a time shift between the $\bar{\eta}_{R}$ process and the wave pressure process: the crest of $\bar{\eta}_{R}$ ahead of the deepest trough occurs at time $-0.45 T_{p}$ and the crest of pressure transducer 6 occurs at time $-0.63 T_{p}$; the crest of $\bar{\eta}_{R}$ following the deepest trough is realized at time $0.42 T_{p}$ and the crest of pressure transducer 6 occurs at time $0.59 T_{p}$.

On the whole, as regard to the mechanics of the interaction of nonlinear three-dimensional sea wave groups with an upright structure, for the case of perfect reflection and without overtopping, the proposed analytical nonlinear theory shows interesting results.

It reveals the presence of characteristic humped wave pressures when the highest wave crest occurs at the structure. A trend, which is not realized with regularly at sea. Anyway, the theory is able to catch and well describe, on one hand the great reduction of the highest crests and the enhancement of the deepest troughs on wave pressures and, on the other hand, the strong asymmetry between the absolute maxima and the minima of the wave pressure profiles, which are distinctive effects of nonlinearity.

Nevertheless, further in-depth studies are required in order to better analyse and to understand the mechanics of generation of all the described phenomenon.

\section{ACKNOWLEDGMENTS}

The present study has been developed with the data of a small scale field experiment conceived and directed by prof. Paolo Boccotti, to whom is the most gratefully acknowledged for the opportunity to attend to his work and to learn from this. 


\section{CONCLUSIONS}

The nonlinear closed-form solution proposed in the paper is able to describe the space-time evolution of three-dimensional sea wave groups propagating in front of an upright structure, for the case of reflection without overtopping. The theory has been applied to evaluate the wave pressure fluctuations in time domain acting on the structure and the relative wave force process, when a very high wave crest, $H_{C R}$, or wave trough, $H_{T R}$, impacts the structure. Theoretical results have been validated through a small-scale field experiment carried out at the Natural Ocean Engineering Laboratory (N.O.E.L., www.noel.unirc.it) of Reggio Calabria (Italy). Records with the occurrence of either the maximum or the minimum wave force recorded during the experiment have been analysed. The comparison between analytical and experimental results has shown a good agreement.

The theory is able to describe the characteristic humped wave pressures, which often occurs at sea when either a high wave crest or a deep wave trough impacts the structure. Anyway, the theory is able to catch and to well describe, the great reduction of the highest crests and the enhancement of the deepest troughs of wave pressures at wall, with respect to linear predictions, and, as a consequence, the strong asymmetry between the absolute maxima and the minima of the wave pressure profiles, which are distinctive effects of non-linearity.

\section{REFERENCES}

Boccotti P., 1981. On the Highest Waves in a Stationary Gaussian Process. Trans. National Ligure Academy of Science and Literature, Genoa, 38, 271.

Boccotti, P., 1982. On Ocean Waves with High Crests. Meccanica, 17, 16-19.

Boccotti P., 1988. Refraction, Reflection and Diffraction of Irregular Gravity Waves, Excepta of Italian Contribution to the Field of Hydraulic Engineering, 3, 47-89.

Boccotti, P., 1989. On Mechanics of Irregular Gravity Waves. Atti Acc. Naz. Lincei, Memorie, VIII, 19, 111-170.

Boccotti P., Barbaro G. and Mannino L., 1993a. A Field Experiment on the Mechanics of Irregular Gravity Waves. $J$. Fluid Mech., 252, 173.

Boccotti P., Barbaro G., Fiamma V.. Mannino L. and Rotta A., 1993b. An Experiment at Sea on the Reflection of the Wind Waves, Ocean Engineering, 20, 493.

Boccotti P., 1997. A General Theory of Three-Dimensional Wave Groups. Part I: The Formal Derivation. Part II: Interaction with a Breakwater, Ocean Engineering, 24, 265-300.

Boccotti P., 2000. Wave Mechanics for Ocean Engineering, Elsevier Science, New York, 496.

Boccotti, P., Arena, F., Fiamma, V., Romolo, A. \& Barbaro, G., 2010. A small scale field experiment on wave forces on upright breakwaters, under peer review.

Goda Y., 1967. The Fourth Order Approximation to the Pressure of Standing Waves, Coastal Engineering in Japan, 10, $1-11$.

Goda Y., 1999. Random Seas and Design in Maritime Structures, World Scientific.

Jeng D. S. 2002. Wave Kinematics of Partial Reflection from a Vertical Wall. Ocean Engineering, 29, 1711.

Hasselmann K., Barnett T. P., Bouws E., Carlson H., Cartwright D. E., Enke K., Ewing J. A., Gienapp H., Hasselmann D. E., Krusemann P., Meerburg A., Müller P., Olbers D. J., Richter K., Sell W., and Walden H., 1973. Measurements of wind wave growth and swell decay during the Joint North Sea Wave Project JONSWAP, Dtsch. Hydrogr. Z. A8, 1.

Kimmoun O., Branger H. and Kharif C., 1999. On Short-Crested Waves: Experimental and Analytical Investigation. European Journal of Mechanics B. Fluids, 18, 889 .

Lin M. C., Liu S. J. and Chuang M. F., 1987. Numerical Analysis of the Short-Crested Water Wave Properties. J. Soc. Naval Arch. Marine Eng, ROC 6, 1, 269.

Longuet-Higgins M. S. and Dommermuth D. G., 2001. On the Breaking of Standing Waves by Falling Jets. Phys. Fluids, 13,1652 .

Longuet-Higgins M. S. and Dommermuth D. G., 2001. Vertical Jets from Standing Waves. II. Proc. R. Soc. Lond., 457, 2137.

Longuet-Higgins M. S. and Drazen D. A., 2002. On Steep Gravity Waves Meeting a Vertical Wall: a Triple Instability. $J$. Fluid Mech., 466, 305.

Marchant T. R. \& Roberts A. J. 1987. Properties of short-crested waves in water of finite depth. J. Aust. Math. Soc. B, 29 , 103.

Mercer G. N. and Roberts A. J., 1992. Standing Waves in Deep Water: Their Stability and Extreme Form. Phys. Fluids, $4,259$.

Mercer G. N. and Roberts A. J., 1994. The Form of Standing Waves on Finite Depth Water. Wave Motion, 19, 233.

Mitsuyasu, H., Tasai, F., Suara, T., 1975. Observation of directional spectrum of ocean waves using a clover-leaf buoy. Journal of Physical Oceanography 5, 750-760.

Peregrine D. H., 2003. Water-wave impact on walls. Annual Review of Fluid Mechanics, 35, 23-43. doi: 10.1146/annurev.fluid.35.101101.161153

Prabhakar V. and Sundar V., 2001. Standing Wave Pressures on Walls. Ocean Engineering, 28, 439.

Roberts A. J. 1983a. A stable and accurate numerical method to calculate the motion of a sharp interface between fluids. IMA J. Appl. Math., 31, 13. 

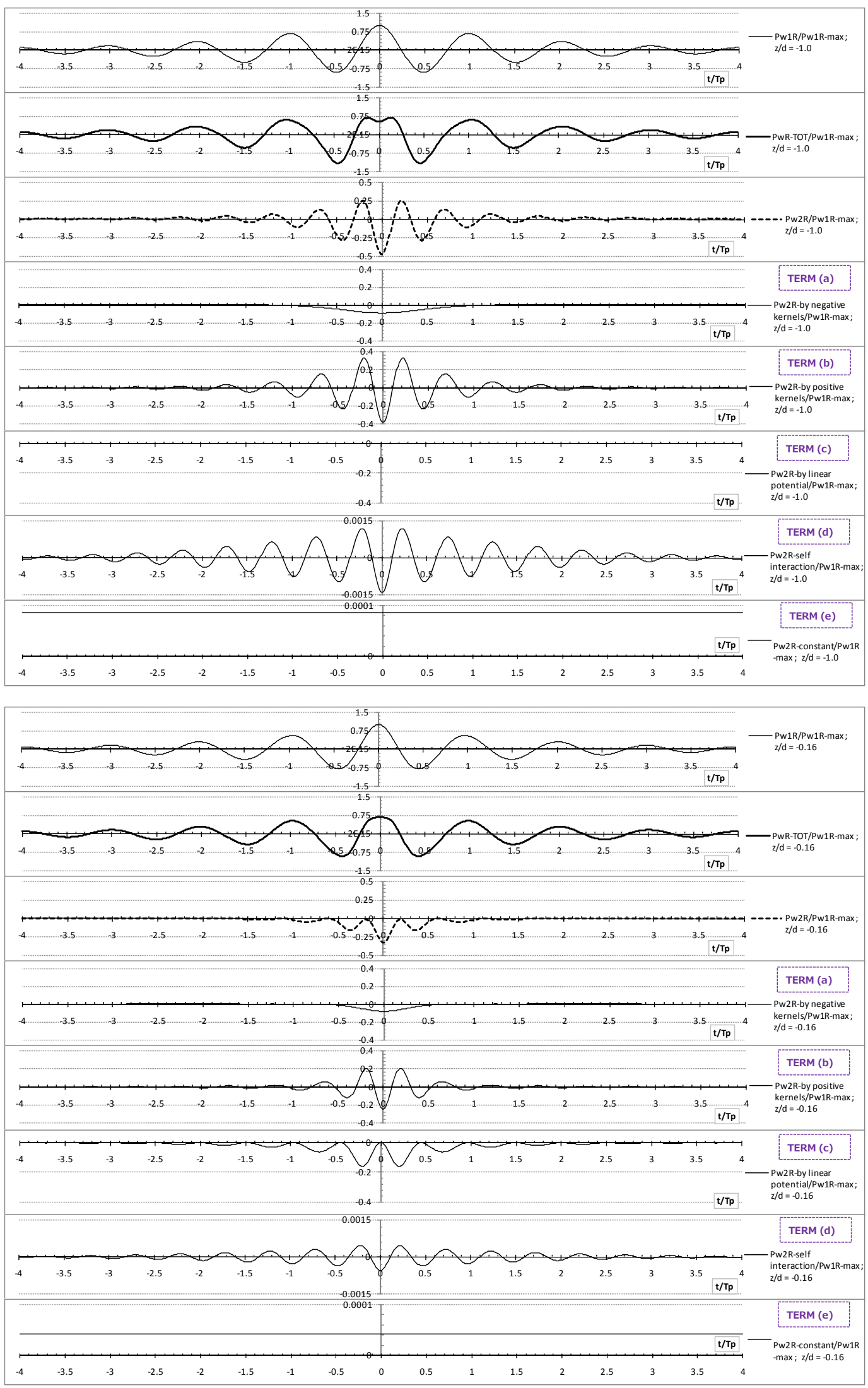

Figure 4. Theoretical linear and nonlinear fluctuating wave pressures acting on the reflective sea wall, when an exceptionally high crest elevation $H_{C R}$ is realized at time instant $T=0$ on the structure. The trends of all the terms defining the second-order components versus time are shown. (Note that the scale in ordinate are different). 

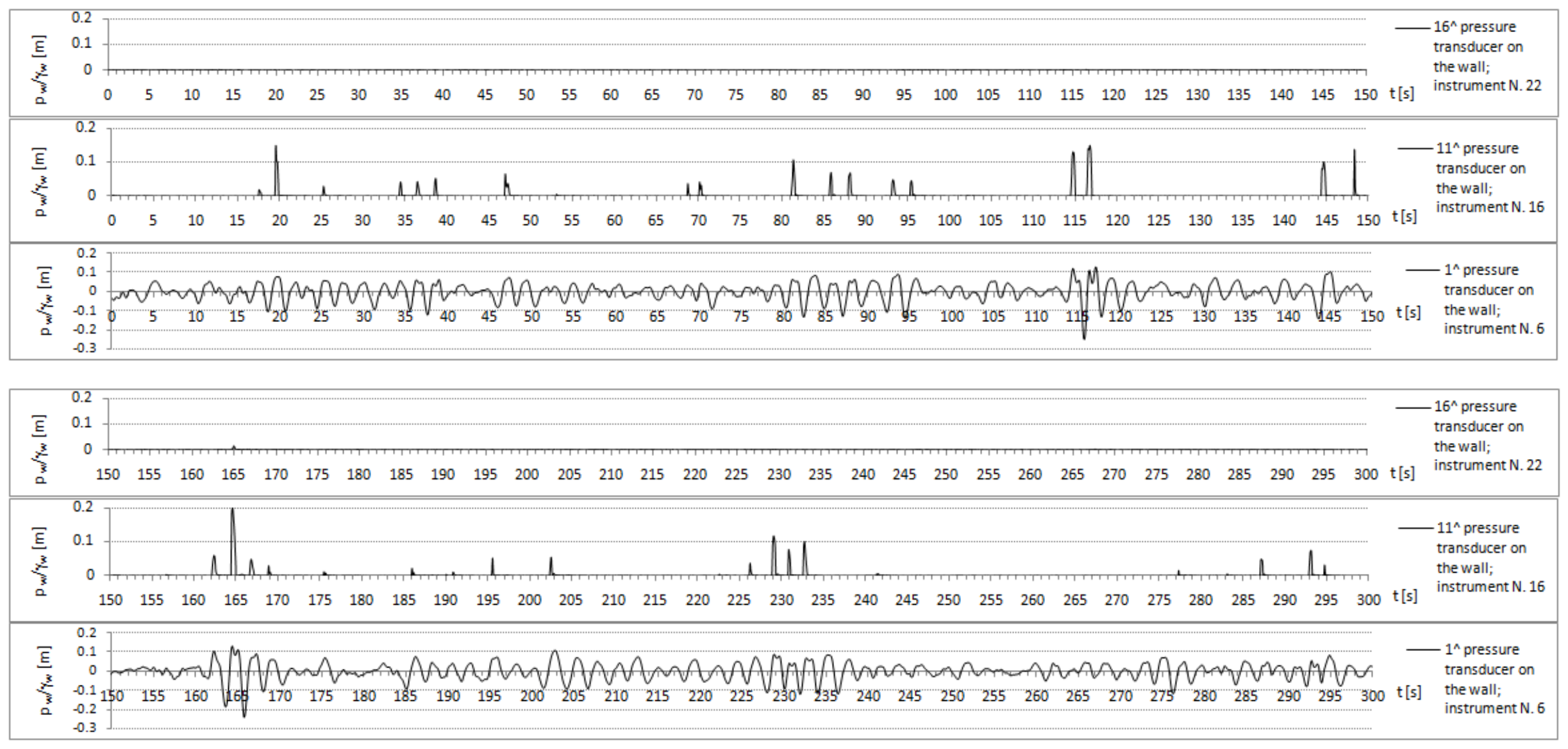

Figure 5. RECORD 951 where the maximum $F_{w}{ }^{-}$occurs, which is also the maximum $F_{w}$ in absolute value of the experiment. Time series of the wave pressures at the reflective wall recorded just at the sea. 


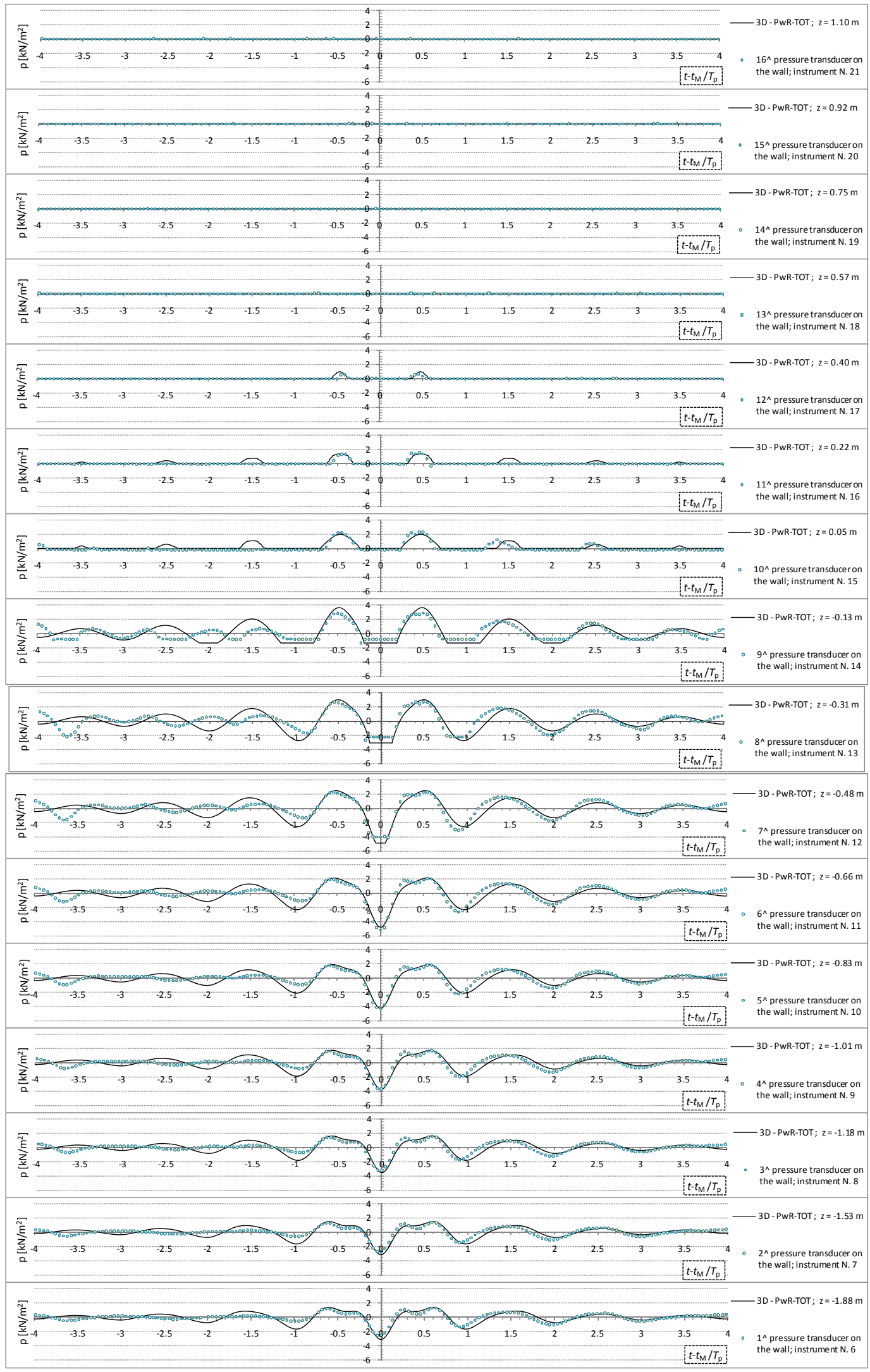

Figure 6. RECORD 951, centred at time instant $t_{\mathrm{M}}$, when the maximum ${F_{w}}^{-}$occurs. That is the maximum $F_{w}$ in absolute value realized during the experiment of May 2009. Points are the data recorded at the sea and the continuous line is obtained by analytical solution, which is applied when an exceptionally linear high trough elevation $H_{T_{1}}\left(=8 \sigma_{\mathrm{und}}\right)$ occurs at the structure at time instant $t / T_{\mathrm{p}}=0$. The minimum of $F_{w}$ has been individualized in the time interval 159s-171s of the record. 


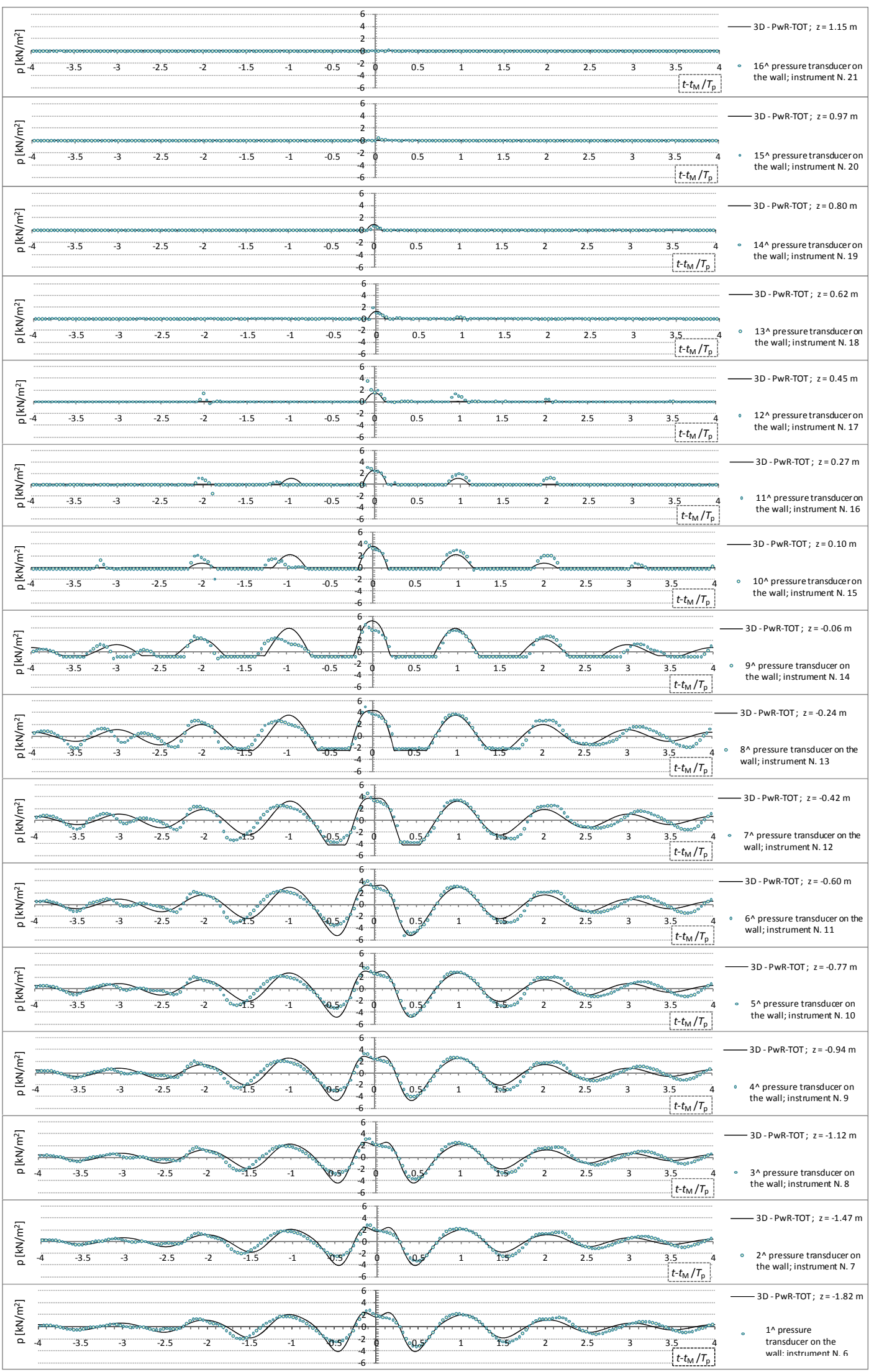

Figure 7. RECORD 1181 centred at time instant $t_{\mathrm{M}}$ when a local theoretical minimum of the wave pressure occurs by considering, with the theory, the occurrence of an exceptionally linear high wave crest elevation $H_{\mathrm{C} 1}\left(=8 \sigma_{\text {und }}\right)$ at the structure at time instant $t / T_{\mathrm{p}}=0$. Theoretical trends are the continuous line, points are the data recorded at the sea. 
Roberts A. J. 1983b. Highly nonlinear short-crested water waves. J. Fluid Mech. 135, 301.

Roberts A. J. and Schwartz L. W., 1983. The Calculation of Nonlinear Short-Crested Gravity Waves. Phys. Fluids. 26, 9 , 2388.

Romolo A., 2007. Mechanics of Nonlinear Random Sea Waves interacting with a Reflective Upright Breakwater. Formal Derivation and Validation' (in English), PhD Thesis on Ocean Engineering at 'Mediterranea' University of Reggio Calabria, n. OE3 - SSD: ICAR/02, 1-291.

Romolo A., Arena F, 2007. Non-linear extreme random forces on a vertical wall given by very high sea wave groups, 5th Coastal Structures International Conference (cst07), 2-4, Luglio, 2007, 5th Coastal Structures International Conference (CSt07) - ASCE., Volume name: Functional Design, Venezia, Italia, Vol. 1, 2009, pp. 895-906.

Romolo, A. \& Arena, F. 2008a. Mechanics of nonlinear random wave groups interacting with a vertical wall, Physics of Fluids. Vol. 20, Issue 3, paper 036604, pp. 1-16. DOI: 10.1063/1.2890474.

Romolo A., Arena F. 2008b. Three-dimensional Nonlinear Wave Groups interacting with an Upright Breakwater. Proc. 31 st International Conference on Coastal Engineering (ICCE 2008) - ASCE. Hamburg, Germany, Aug. 31 - Sept. $5,2008$.

Saintflou G. 1928. Essai sur les digues maritimes verticales. Annual Ponts et Chaussees, 98 (1), 5-48.

Schultz W. W., Vanden-Broeck J.-M., Jiang L. and Perlin M., 1998. Highly Nonlinear Standing Water Waves with Small Capillary effect. J. Fluid Mech., 369, 253.

Svendsen I. A. \& Jonsson I. G. 1976. Hydrodynamics of Coastal Regions. Den Private Ingeniorfond-Technical University of Denmark, DK-2800, Lyngby.

Tadjbakhsh I. \& Keller J. B. 1960. Standing Surface Waves of Finite Amplitude, J. Fluid Mech. 8, 442.

Tsai C. P., Jeng D.S. and Hsu J. R. C., 1994. Computations of the almost Highest Short-Crested Waves in Deep Water. Applied Ocean Research 16, 6, 317.

\section{APPENDIX}

The interaction kernels $\mathrm{A}_{n}^{\mp}$ and $\mathrm{C}_{n}^{\mp}(n=1,2)$ respectively of the nonlinear free surface $\bar{\eta}_{2_{R}}$ and of the velocity potential $\bar{\phi}_{2_{R}}$ are defined as sum and difference of frequencies as follows

$$
\begin{aligned}
& \mathrm{A}_{n}^{-}\left(\omega_{1}, \omega_{2}, \theta_{1}, \theta_{2} ; d\right)=g \omega_{1}^{-1} \omega_{2}^{-1}\left\{\mathrm{~B}_{n}^{-}-k_{1} k_{2}\left[-(-1)^{n} \cos \left(\theta_{1}+(-1)^{n} \theta_{2}\right)\right.\right. \\
& \left.\left.+\tanh \left(k_{1} d\right) \tanh \left(k_{2} d\right)\right]\right\}+\left(\omega_{1}^{2}+\omega_{2}^{2}\right) / g \\
& \mathrm{~A}_{n}^{+}\left(\omega_{1}, \omega_{2}, \theta_{1}, \theta_{2} ; d\right)=g \omega_{1}^{-1} \omega_{2}^{-1}\left\{\mathrm{~B}_{n}^{+}-k_{1} k_{2}\left[-(-1)^{n} \cos \left(\theta_{1}+(-1)^{n} \theta_{2}\right)\right.\right. \\
& \left.\left.-\tanh \left(k_{1} d\right) \tanh \left(k_{2} d\right)\right]\right\}+\left(\omega_{1}^{2}+\omega_{2}^{2}\right) / g \\
& \mathrm{C}_{n}^{-}\left(\omega_{1}, \omega_{2}, \theta_{1}, \theta_{2} ; d\right)=\mathrm{B}_{n}^{-} \frac{\cosh \left[\left|\underline{\mathbf{k}}_{n}^{-}\right|(d+z)\right]}{\cosh \left(\left|\underline{\mathbf{k}}_{n}^{-}\right| d\right)} \quad n=1,2 . \\
& \mathrm{C}_{n}^{+}\left(\omega_{1}, \omega_{2}, \theta_{1}, \theta_{2} ; d\right)=\mathrm{B}_{n}^{+} \frac{\cosh \left[\left|\underline{\mathbf{k}}_{n}^{+}\right|(d+z)\right]}{\cosh \left(\left|\underline{\mathbf{k}}_{n}^{+}\right| d\right)}
\end{aligned}
$$

with the $\mathrm{B}_{n}^{\mp}(n=1,2)$ parameters equal to

$$
\begin{aligned}
& \mathrm{B}_{n}^{-}\left(\omega_{1}, \omega_{2}, \theta_{1}, \theta_{2} ; d\right)=\frac{\left(\omega_{1}-\omega_{2}\right)\left\{\omega_{2} k_{1}^{2}\left[1-\tanh ^{2}\left(k_{1} d\right)\right]-\omega_{1} k_{2}^{2}\left[1-\tanh ^{2}\left(k_{2} d\right)\right]\right\}}{\left(\omega_{1}-\omega_{2}\right)^{2}-g\left|\underline{\mathbf{k}}_{n}^{-}\right| \tanh \left(\left|\underline{\mathbf{k}}_{n}^{-}\right| d\right)} \\
& +\frac{2\left(\omega_{1}-\omega_{2}\right)^{2} k_{1} k_{2}\left\{\tanh \left(k_{1} d\right) \tanh \left(k_{2} d\right)-(-1)^{n} \cos \left[\theta_{1}+(-1)^{n} \theta_{2}\right]\right\}}{\left(\omega_{1}-\omega_{2}\right)^{2}-g\left|\underline{\mathbf{k}}_{n}^{-}\right| \tanh \left(\left|\underline{\mathbf{k}}_{n}^{-}\right| d\right)} \quad n=1,2 . \\
& \mathrm{B}_{n}^{+}\left(\omega_{1}, \omega_{2}, \theta_{1}, \theta_{2} ; d\right)=\frac{\left(\omega_{1}+\omega_{2}\right)\left\{\omega_{2} k_{1}^{2}\left[1-\tanh ^{2}\left(k_{1} d\right)\right]+\omega_{1} k_{2}^{2}\left[1-\tanh ^{2}\left(k_{2} d\right)\right]\right\}}{\left(\omega_{1}+\omega_{2}\right)^{2}-g\left|\underline{\mathbf{k}}_{n}^{+}\right| \tanh \left(\left|\underline{\mathbf{k}}_{n}^{+}\right| d\right)} \\
& -\frac{2\left(\omega_{1}+\omega_{2}\right)^{2} k_{1} k_{2}\left\{\tanh \left(k_{1} d\right) \tanh \left(k_{2} d\right)+(-1)^{n} \cos \left[\theta_{1}+(-1)^{n} \theta_{2}\right]\right\}}{\left(\omega_{1}+\omega_{2}\right)^{2}-g\left|\underline{\mathbf{k}}_{n}^{+}\right| \tanh \left(\left|\underline{\mathbf{k}}_{n}^{+}\right| d\right)}
\end{aligned}
$$

being

$$
\begin{aligned}
& \underline{\mathbf{k}}_{n}^{-}\left(\omega_{1}, \omega_{2}, \theta_{1}, \theta_{2} ; d\right)=\left(k_{1} \sin \theta_{1}-k_{2} \sin \theta_{2} ; k_{1} \cos \theta_{1}+(-1)^{n} k_{2} \sin \theta_{2}\right) \quad n=1,2 \\
& \underline{\mathbf{k}}_{n}^{+}\left(\omega_{1}, \omega_{2}, \theta_{1}, \theta_{2} ; d\right)=\left(k_{1} \sin \theta_{1}+k_{2} \sin \theta_{2} ; k_{1} \cos \theta_{1}-(-1)^{n} k_{2} \sin \theta_{2}\right)
\end{aligned}
$$

the vectors of wave numbers. 